\title{
FKM Based Approach for Hybrid Video Segmentation
}

\author{
K. Mahesh ${ }^{1}$ and Dr.K. Kuppusamy ${ }^{2}$ \\ ${ }^{1}$ Associate Professor,Department of Computer Sci and Engg \\ Alagappa University, Karaikudi. Tamilnadu India \\ mahesh.alagappa@gmail.com \\ ${ }^{2}$ Associate Professor,Department of Computer Sci and Engg \\ Alagappa University, Karaikudi. Tamilnadu India \\ kkdiksamy@yahoo.com
}

\begin{abstract}
In digital storage media, Video segmentation is one of the popular and important techniques.The challenging problem in digital storage media is video segmentation. In this hybrid video segmentation technique, initially similar shots are segmented. Then subsequently the track frames in every shot are assorted using the extracted objects of every frame which highly mostly reduces the processing time. Here it yields the effective video segmentation results by performing intersection on the segmented results provided by both the frame difference method as well as consecutive frame intersection method. In frame difference method, it considers the key frame as background and it segments the dynamic objects whereas in the frame difference method, it segments the static and dynamic objects by intersection of objects in consecutive frames. This new hybrid video segmentation technique is developed using FKM clustering approach and is evaluated by varying video sequences and then efficiency is analyzed by calculating the statistical measures and kappa coefficient.
\end{abstract}

\section{KEYWORDS:}

Video segmentation, Discrete cosine transform, FKM clustering, Frame difference algorithm, Frame intersection algorithm Euclidean distance, Kappa coefficient.

\section{INTRODUCTION}

The primary aspect of video indexing is it has the potential to segment the video into consequential or meaningful segments [1]. Video applications such as surveillance, communications and entertainment are very crucial for industries. Among these applications, segmentation of moving object is one of the basic operations for several automated video analysis tasks [15]. One of the main challenges in computer vision is automatic comprehension of complex dynamic content of videos, such as detection, localization, and segmentation of objects and people, and understanding their interactions [9]. Image and video segmentation is very beneficial in several applications for finding the regions of interest in a panorama or annotating the data [11]. MPEG-4 is a promising standard for multimedia communications. MPEG-4 
The International Journal of Multimedia \& Its Applications (IJMA) Vol.4, No.4, August 2012

provides standardized ways to encode the video and audio objects, and the scene description, which represents how the objects are structured in a scene [4].

The video segmentation is an imperative technique used for the improvement of video quality on the basis of segmentation [8]. The function of video segmentation is to segment the moving objects in video sequences [19]. Video segmentation is an imperative component of numerous video analysis and coding problems, such as 1) video summarization, indexing, and retrieval, 2) advanced video coding, 3) video authoring and editing, 4) enhanced motion (optical flow) estimation, 5) 3D motion and structure assessment with several moving objects [16]. The structure of the paper is organized as follows: A brief review of the researches related to the video segmentation is given in Section 2. The proposed video segmentation technique is given in section 3. The experimental results of the proposed approach are presented in Section 4. Finally, the conclusions are given in Section 5.

\section{RELATED WORK}

Cheng Chen and Guoliang Fan have proposed a perception principles guided video segmentation method, where statistical modeling and graph-theoretic approaches are combined in multi-layer classification architecture. Various visual cues are effectively incorporated in a sequential segmentation process [16].

Yong Jae Lee,Jaechul Kim, and Kristen Grauman have proposed a technique, discover and segment foreground object(s) in video. Given an unannotated video sequence, the method first identifies object-like regions in any frame according to both static and dynamic cues. We then compute a series of binary partitions among those candidate "key-segments" to discover hypothesis groups with persistent appearance and motion [17].

Yasira Beevi P and S. Natarajan [1] have proposed a video segmentation algorithm for MPEG-4 camera system by means of change detection, background registration methods and real time adaptive threshold techniques. The proposed algorithm has given an acceptable segmentation results with low computation complexity. As well, it has a shadow cancellation mode, which can handle both light changing effect and shadow effect. Moreover, the algorithm has also applied real time adaptive threshold techniques through which the parameters can be determined automatically.

\section{DYNAMIC AND STATIC FOREGROUND SEGMENTATION USING HYBRID TECHNIQUE}

The proposed technique segments both the dynamic and static foreground objects without considering the global motion constraints. The motion segmentation process is carried out by both the frame difference algorithm and intersection method subsequently the most common and accurate segmented objects are retrieved from both the segmented results whereas the static foreground are segmented using the intersection of consecutive frames. 
The International Journal of Multimedia \& Its Applications (IJMA) Vol.4, No.4, August 2012

\subsection{SHOT SEGMENTATION}

The term video commonly refers to several storage formats for storing moving pictures. The video consists of consequent sequence of frames which is a single picture or still shot run in succession to produce what appears to be seamless piece of film or video tape. Let be the video to be segmented where ' $L$ ' is the total no of frames present in the video. A shot is defined as a sequence of frames which are captured from a single camera operation. Prior to video segmentation, the shot segmentation is necessary for grouping the similar shots. In this shot segmentation similar shots are grouped together for improving the performance of the segmentation. To accomplish this task initially all the frames are partitioned into $m \times n$ patches and every patch are converted to its equivalent frequency coefficients by means of Discrete Cosine Transform (DCT) developed by Ahmed, Natarajan and Rao (1974) (i.e) DCT is applied to every patch in the frames as follows

$T_{i k}=D_{i k} \times f_{i k} \times D^{\prime}$

Where

$\eta_{a}= \begin{cases}(1 / 2)^{1 / 2} & \text { if } \quad a=0 \\ 1 & \text { if } 1 \leq c \leq m-1\end{cases}$
$\alpha_{c}= \begin{cases}(1 / 2)^{1 / 2} & \text { if } \quad b=0 \\ 1 & \text { if } 1 \leq c \leq n-1\end{cases}$

$1 \leq k \leq P_{(i)}$ where $P_{i}$ is the number of patches present in the $\mathrm{i}^{\text {th }}$ frame. Thus all the patches are transformed to transform domain subsequently the Euclidean distance of every patches of consequent frames and their total mean are calculated as follows.

$$
D_{j}=\frac{\sum_{i=1 ; k=1}^{L ; P_{i}} \sqrt{\left(T_{i, k}-T_{i+1, k}\right)^{2}}}{P_{(i)}}
$$

Where $1<i, j \leq L$ and $1 \leq k \leq P_{(i)}$. The frames belongs to the similar shots are identified based on the mean distance. If $D_{j} \leq E \quad f_{i}$ and $f_{i+1}$ belong to the same shots otherwise they are belong to the different shots.

\subsection{OBJECT EXTRACTION}

Initially the objects in every frame are identified for segmentation. 
The International Journal of Multimedia \& Its Applications (IJMA) Vol.4, No.4, August 2012

Let $\delta=\left\{\delta_{a} \mid 1<a \leq \mathbf{A}\right\}$ be the result of shot segmentation where 'A' is total no of shots and $\delta_{a}=\left\{f_{a j}|1<a \leq \mathbf{A} ; 1<j \leq| \delta_{a} \mid\right\}$ be the set of similar shots where ' $\left|\delta_{a}\right|$ ' are the total no of frames in $\mathrm{a}^{\text {th }}$ shot in the segmented results. The initial frames in every shot are taken as key frame for object extraction for example the $f_{11}$ is key frame for shot $\delta_{1}$ which is known as $f_{k e y(1)}$. Like wise each shot having its own key frames. Initially all the frames which are in RGB color format are converted to grey scale format. A RGB color is another format for color images and it represents an image with three matrices of sizes matching the image format while each matrix corresponds to one of the colors red, green and blue. [26] When we convert it into a grey scale (or "intensity") image, it depends on the sensitivity response curve of detector to light as a function of wavelength [27]. This conversion process yields grey scale image.

The objects are identified by the clustering process which is carried out using fuzzy k-means clustering. The value of ' $\mathrm{k}$ ' can be identified easily for static single image but since the video images are dynamic in nature, the $3 \mathrm{~d}$ color histogram is calculated for every frame to find the no of objects in a frame. Before calculating 3D histogram value the frames in 'RGB' format is converted into $\mathrm{LAB}$ color space. The number of peaks values in the $3 \mathrm{D}$ color histogram determines the total no of objects in a frame which is given as the value of ' $\mathrm{k}$ ' in fuzzy k-means clustering. Finally the clustering process yields the no of objects in a frame. The overlapping objects are also identified using fuzzy k-means clustering.

\section{Fuzzy k-means clustering Algorithm}

Input: gray scale converted frames $\hat{f}_{a j}$ of the shot $\delta_{a}$

Output: The Resultant Solution Clusters.

\section{Procedure:}

1) For each $\hat{f}_{a j} \in \delta_{a}$

2) Make initial guesses for the means $m 1, m 2 \ldots m k$

3) Until there are no changes in mean.

4) Use the estimated means to find the degree of Membership $\varpi_{i j}$ of $\hat{f}_{a j_{i j}}$ in cluster I.

5) For $\mathrm{I}=1$ to $\mathrm{K}$

6) Replace $m i$ with the fuzzy mean of all of the examples for cluster i.

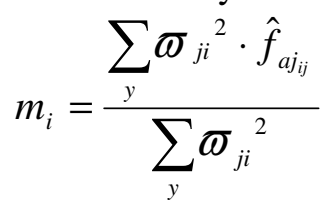

7) End For

8) End Until

9) End For 
The International Journal of Multimedia \& Its Applications (IJMA) Vol.4, No.4, August 2012

\subsection{TRACK FRAME ASSORTMENT}

After performing shot segmentation, the track frames of the every shot are identified using the objects of their key frame. The objects that appear simultaneously in at least two consecutive frames can be compared directly in terms of their motion so the assortment of the track frames is a required preprocessing step for segmentation this track frame selection process reduces the computational time of segmentation. The objects of the key frame are compared with the other frames of the shot for their presence in the frame. If the object is present in any of the frame then its frame index is stored in $T_{j}{ }^{i}$. For example if the object of key frame $f_{\text {key }(1)}$ is presented in the $\mathrm{k}^{\text {th }}$ index of shot then the index is stored in $T_{1}^{1}$. Like wise all the track frames of the every shot are identified.

\subsection{DYNAMIC FOREGROUND SEGMENTATION BASED ON FRAME DIFFERENCE ALGORITHM}

In the background subtraction method the key frame of every shot is consider as background.At each $\hat{f}_{a j}$ frame, the $\hat{f}_{a j}(p, q)$ pixel's value can be classified as foreground pixel if the following inequality

$\hat{f}_{a j}(p, q)-\hat{f}_{a 1}(p, q)>\lambda$

Holds; otherwise $\hat{f}_{a j}(p, q)$ will be classified as background pixel value. Where $\hat{f}_{a j}(p, q)$ is the current frame pixel value, $\hat{f}_{a 1}(p, q)$ is the key frame value and ' $\lambda$ ' is the threshold pixel value in foreground.

\subsection{STATIC AND DYNAMIC FOREGROUND SEGMENTATION USING INTERSECTION OF FRAMES}

The motion analysis and segmentation of dynamic objects is performed by intersection process of track frames. Initially the frames in every shot are converted to binary form.

\subsubsection{BINARIZATION}

Binarization is a technique by which the gray scale images are converted to binary images. Binarization separates the foreground (text) and background information. The most common method for binarization is to select a proper threshold for the intensity of the image and then convert all the intensity values above the threshold to one intensity value ("white"), and all intensity values below the threshold to the other chosen intensity ("black"). 
$\hat{\hat{f}}_{a j}=\left\{\begin{array}{l}1 ; \text { if } \hat{f}_{a j}>\Gamma \\ 0 ; \text { otherwise }\end{array}\right\}$

Where ' $\Gamma$ ' is a global threshold value for binarization. After performing the binarization the consecutive frames are intersected to segment the dynamic and static objects.

Let $\hat{\hat{f}}_{11}$ and $\hat{\hat{f}}_{12}$ be the binarized form of frame 1 and frame 2 in shot 1 respectively. The dynamic motion objects are found as follows

$G 2_{a j}=\hat{\hat{f}}_{11}-f_{a j}^{\prime}$

Whereas the static foreground are segmented as follows

$$
f_{a j}^{i}=\hat{\hat{f}}_{11} \cap \hat{\hat{f}}_{12}
$$

Like wise all the consecutive frames are intersected to achieve the static and dynamic object segmentation.

\subsection{HYBRIDIZATION OF SEGMENTATION METHODS}

Let $G 1=\left\{S_{i} \mid 1<i \leq n\right\}$ and $G 2=\left\{S_{i}^{\prime} \mid 1<i \leq n\right\}$ be the segmented results of dynamic objects using frame difference algorithm and frame intersection method respectively. The prior said technique yields the segmented motion objects by subtracting the background and the later segmentation technique yields the motion object by intersection method. The ' $G$ 'consists of final segmented motion objects.

$$
G=G 1 \cap G 2
$$

\section{EXPERIMENTAL ANALYSIS}

Our new hybrid video segmentation approach has been validated by experimenting with variety of video sequences. This hybrid video segmentation technique yields the dynamic object segmentation results by intersection of segmented results of both the frame difference algorithm and intersection methods and hence produces the better enhanced segmented results. Also the proposed system segments the static objects in every frame. It represents the dynamic object segmentation results by using frame difference algorithm which considers the key frame of every shot as background. It illustrates the segmented results of intersection methods which contains both the segmented results of static and dynamic objects. It represents the final video segmented results of the proposed hybrid segmentation technique. In the green color represents the dynamic objects and the other color represents the static objects. 
The International Journal of Multimedia \& Its Applications (IJMA) Vol.4, No.4, August 2012

\section{1. EXPERIMENTAL RESULTS}

Our hybrid video segmentation approach has been validated by experimenting with variety of video sequences. The proposed system has been implemented in Matlab (Matlab7.10).

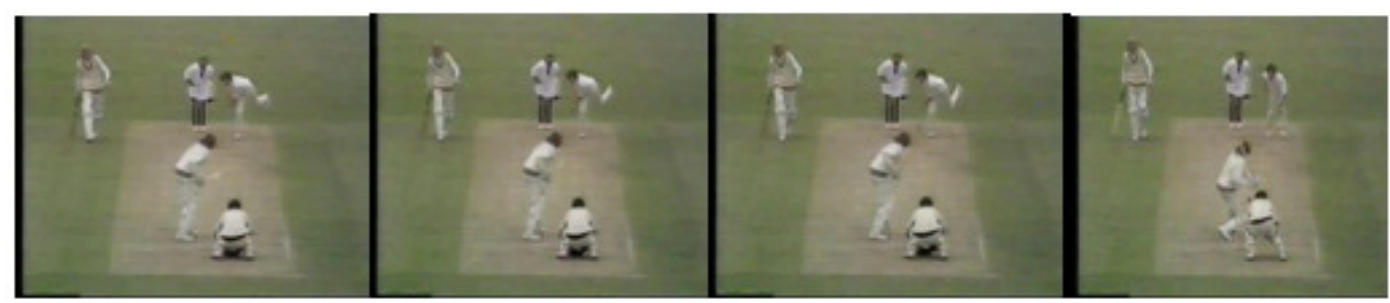

(a)

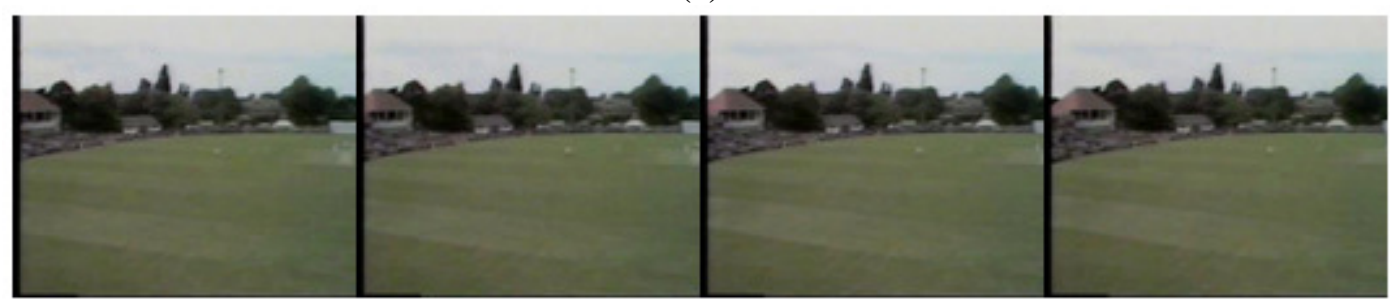

(b)

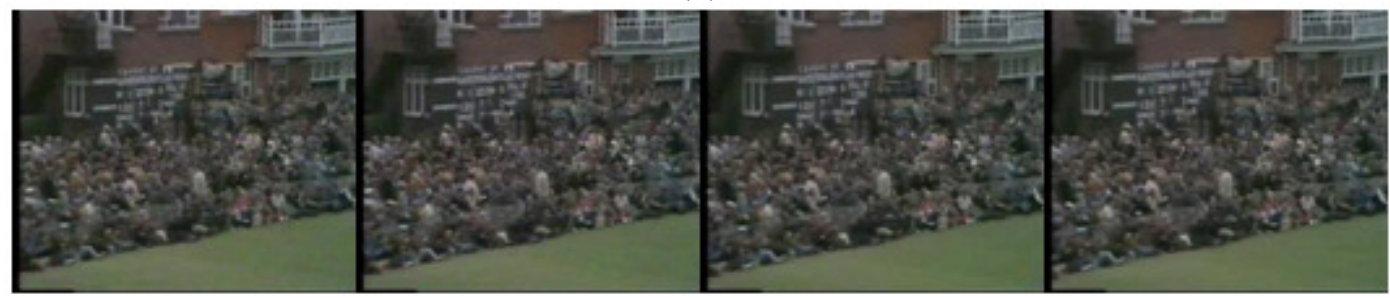

(c)

Figure 3: Sample similar shot segmented results

Initially the similar shots are grouped together by patch processing method. The fig. 3(a), (b), (c) represents the sample results of shot segmentation process.

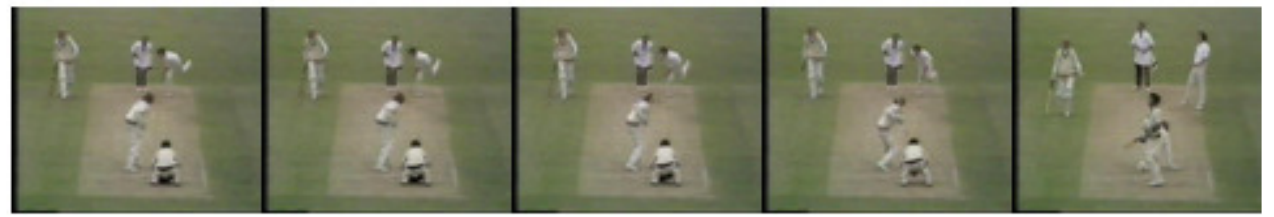

(a)

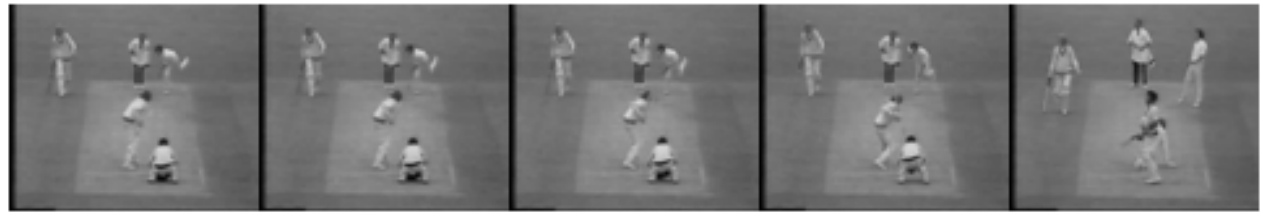

(b) 
The International Journal of Multimedia \& Its Applications (IJMA) Vol.4, No.4, August 2012

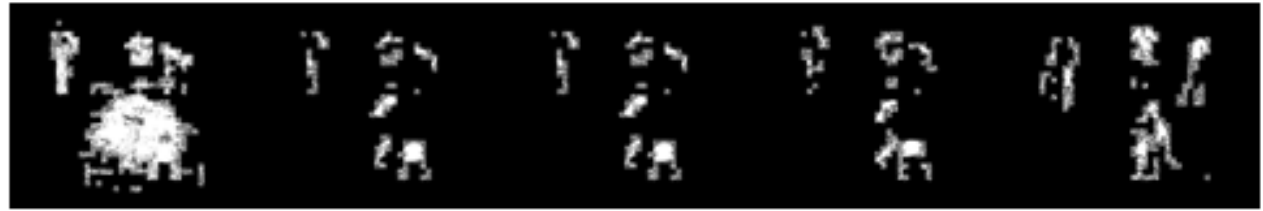

(c)

Figure 4: (a) Sample frames, (b) Grayscale images, (c) Extracted Objects

The fig. 4(a) represents the sample frames(Frame :2,Frame :3, Frame :4, Frame :5, Frame :44) in a tested video and the fig. 4(b),4(c) represents their corresponding gray scale converted image .Considering the initial frame as key frame all the objects in the every frames are extracted. The fig. 4(c) represents the extracted objects of the frames in fig. 4(a).
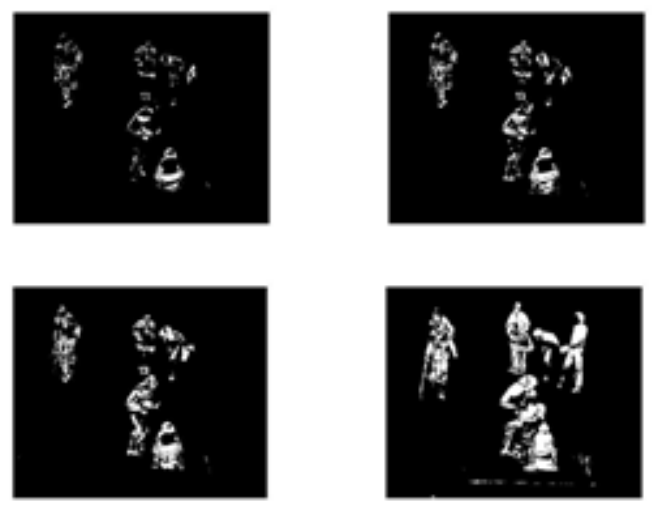

Figure 5: Segmented dynamic objects using frame difference algorithm

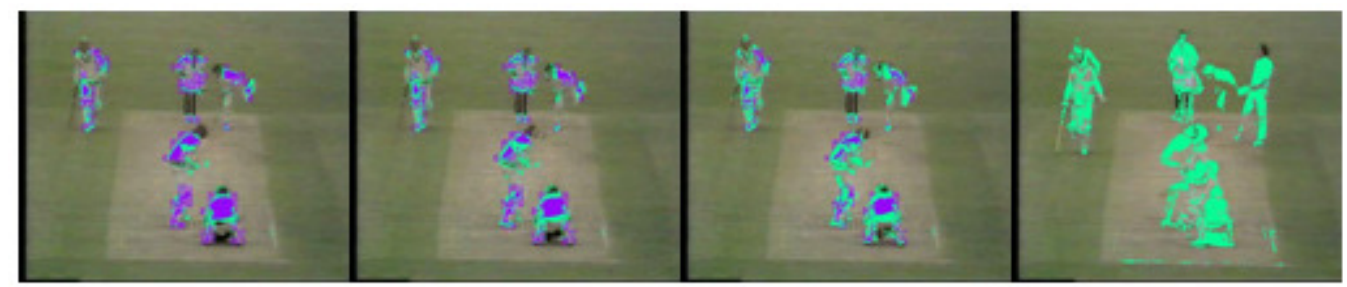

Figure 6: Segmented static and dynamic objects using intersection method

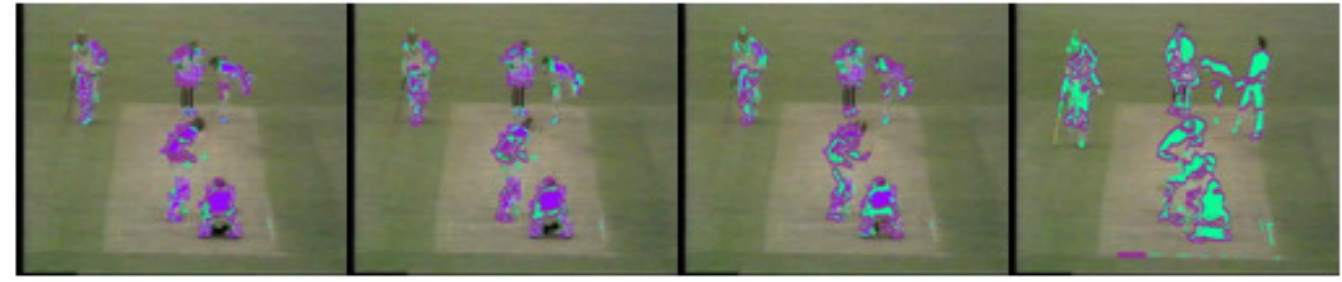

Figure 7: Segmented static and dynamic objects using proposed hybrid method

\subsection{Performance Evaluation}

The performance of the proposed system is evaluated by the statistical measures like sensitivity and specificity. The output of the proposed system may be positive (Segmenting the objects) or 
The International Journal of Multimedia \& Its Applications (IJMA) Vol.4, No.4, August 2012

negative (non-segmenting the objects). The output of the proposed system may or may not be match with the original status of the image. Consider the following setting for the statistical measures.

True Positive (TP) : Valid objects correctly segmented.

False Positive (FP) : Invalid objects incorrectly segmented.

True Negative (TN) : Invalid objects correctly non-segmented.

False Negative (FN) : Valid objects incorrectly non-segmented

Sensitivity value represents the percentage of recognition of actual values. Specificity value represents the percentage of recognition of actual negatives. Accuracy is degree of closeness of measurements of a quantity to its actual (true) value. The performance is also analysis by the kappa coefficient which is as below. It represents the statistical measures of the proposed system for the different frames in a video sequence-I

\subsection{COMPARATIVE ANALYSIS}

The performance of the proposed hybrid segmentation technique is also evaluated by comparing its segmented results with that of the traditional video segmentation technique which uses background substraction method.

The fig. 10(a) represents the sample frames (Frame: 23, Frame: 24, Frame: 25 and Frame: 65) of a video sequence. The fig. 10(b) and (c) represents the video segmentation results of the frames in fig. 10(a) using the proposed system and by using the conventional segmentation technique respectively. The table- 3 and table- 4 represents the comparison statistical measures of the segmentation of video-II using the proposed technique as well as the conventional method and the fig.11 illustrates the corresponding accuracy comparison graph.

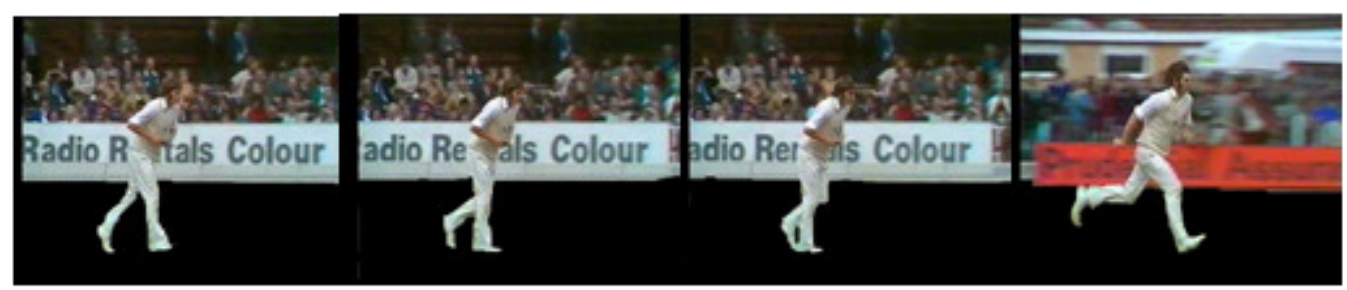

(a)

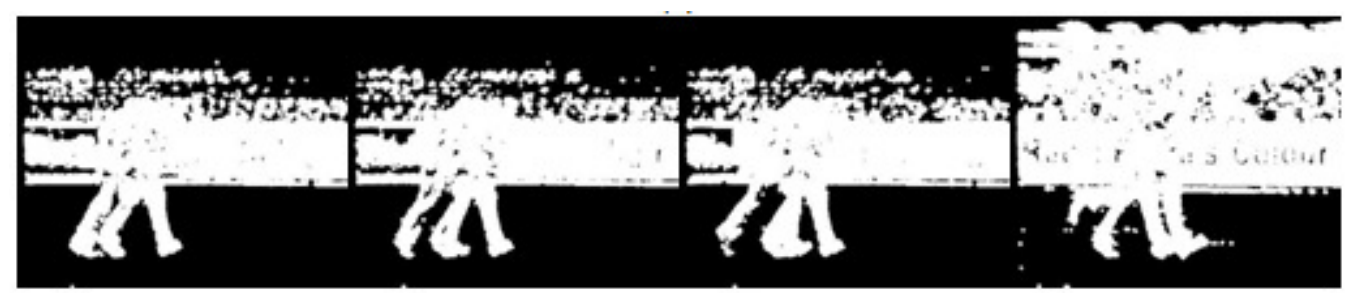

(b) 
The International Journal of Multimedia \& Its Applications (IJMA) Vol.4, No.4, August 2012

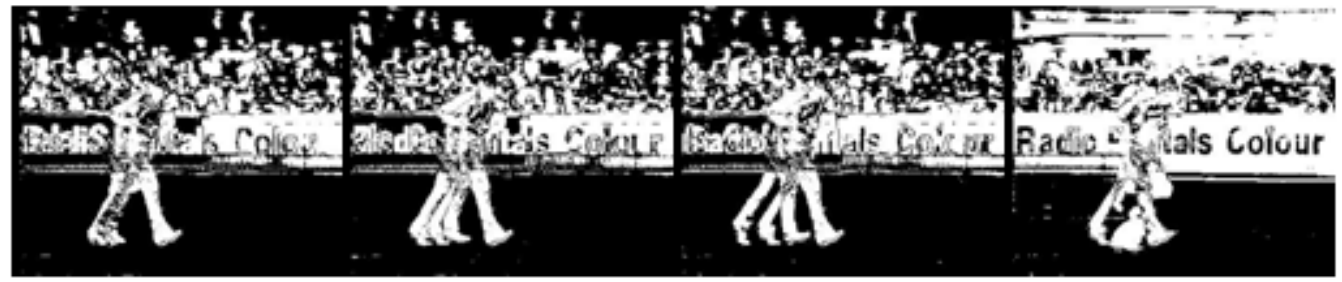

(c)

Figure 10: (a) Sample Frames (b) Segmented Results using proposed system (c) Segmented results using conventional segmentation technique

Table3: Statistical Measures of the proposed System for Video-II using the proposed system

\begin{tabular}{|l|c|c|c|c|}
\hline \multicolumn{1}{|c|}{ Measures } & Frame23 & Frame24 & Frame25 & Frame65 \\
\hline TP & 30784 & 29747 & 30724 & 54880 \\
\hline TN & 59180 & 59944 & 59409 & 40170 \\
\hline FP & 10897 & 11685 & 11243 & 6326 \\
\hline FN & 6006 & 6916 & 6547 & 7888 \\
\hline TPR or Sensitivity & 83.67491 & 81.1363 & 82.43406 & 87.43309 \\
\hline FPR & 15.55 & 16.31 & 15.91 & 13.61 \\
\hline Accuracy & 84.18 & 82.82 & 83.52 & 86.99 \\
\hline $\begin{array}{l}\text { Specificity or True } \\
\text { Negative rate }\end{array}$ & 84.45 & 83.69 & 84.09 & 86.39 \\
\hline $\begin{array}{l}\text { Positive Predictive } \\
\text { value }\end{array}$ & 73.86 & 71.8 & 73.21 & 89.66 \\
\hline $\begin{array}{l}\text { Negative Predictive } \\
\text { value(NPV) }\end{array}$ & 90.79 & 89.66 & 90.07 & 83.59 \\
\hline False discovery rate & 26.14 & 28.2 & 26.79 & 10.34 \\
\hline $\begin{array}{l}\text { Mathews Correlation } \\
\text { Coefficient }\end{array}$ & 0.66 & 0.63 & 0.65 & 0.74 \\
\hline Kappa Coefficient & 0.70616 & .0 .712265 & 0.708688 & 0.677854 \\
\hline
\end{tabular}

Table 4: Statistical Measures of the proposed System for Video-II using the conventional frame difference method

\begin{tabular}{|l|c|c|c|c|}
\hline \multicolumn{1}{|c|}{ Measures } & Frame23 & Frame24 & Frame25 & Frame65 \\
\hline TP & 23241 & 22644 & 23952 & 44073 \\
\hline TN & 59180 & 59944 & 59409 & 40170 \\
\hline FP & 18440 & 18788 & 180155 & 17133 \\
\hline FN & 6256 & 8416 & 8057 & 6487 \\
\hline TPR or Sensitivity & 78.79106 & 72.90406 & 74.82895 & 87.1697 \\
\hline FPR & 23.76 & 23.86 & 75.2 & 29.9 \\
\hline Accuracy & 76.94 & 75.22 & 30.7 & 78.1 \\
\hline $\begin{array}{l}\text { Specificity or True } \\
\text { Negative rate }\end{array}$ & 76.24 & 76.14 & 24.8 & 70.1 \\
\hline
\end{tabular}


The International Journal of Multimedia \& Its Applications (IJMA) Vol.4, No.4, August 2012

\begin{tabular}{|l|c|c|c|c|}
\hline $\begin{array}{l}\text { Positive Predictive } \\
\text { value }\end{array}$ & 55.76 & 54.65 & 11.74 & 72.01 \\
\hline $\begin{array}{l}\text { Negative Predictive } \\
\text { value(NPV) }\end{array}$ & 90.44 & 87.69 & 88.06 & 86.1 \\
\hline False discovery rate & 44.24 & 45.35 & 88.26 & 27.99 \\
\hline $\begin{array}{l}\text { Mathews Correlation } \\
\text { Coefficient }\end{array}$ & 0.5 & 0.46 & 0 & 0.58 \\
\hline Kappa Coefficient & 0.660146 & .0 .676096 & 0.674019 & 0.489013 \\
\hline
\end{tabular}

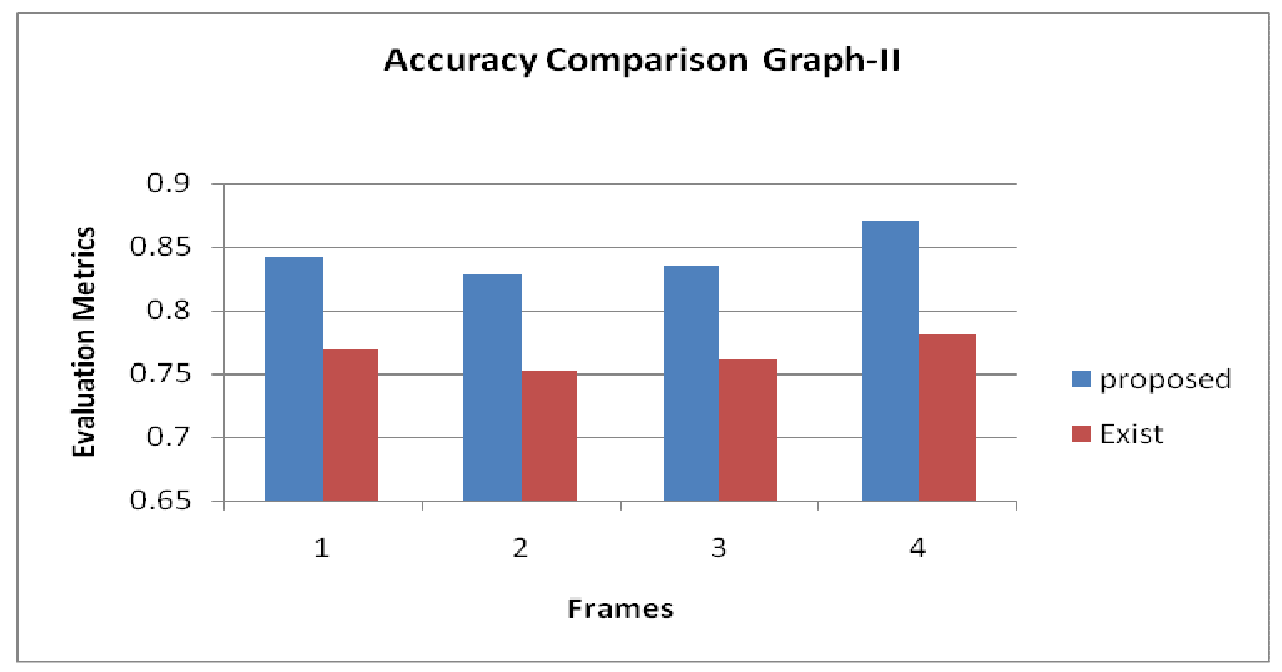

Figure 11: Accuracy Comparison Graph-II

\section{CONCLUSION}

In this paper, we have proposed a new hybrid video segmentation technique to segment both the static and dynamic objects. This is intended to overcome the existing frame difference based segmentation techniques. The segmentation technique based on frame difference algorithm segmented the objects by considering the key frame as background which only produced the motion difference from key frame with remaining frames but the proposed technique also considered the consecutive frame differences by using the consecutive frame intersection method and hence provided better results. The comparison shows the effective accuracy of the proposed technique and also the proposed technique achieve $88 \%$ accuracy. The accuracy is evaluated by different video sequences.

\section{References}

[1] "A Hidden Markov Model Framework for Video Segmentation Using Audio and Image Features" citeseerx.ist.psu.edu/viewdoc/download?doi=10.1.1.20...rep. 
The International Journal of Multimedia \& Its Applications (IJMA) Vol.4, No.4, August 2012

[2] Panagiotis Sidiropoulos1, Vasileios Mezaris, Ioannis Kompatsiaris, Hugo Meinedo, Miguel Bugalho, Isabel Trancoso,"Video scene segmentation system using audio visual features", Workshop on Image Analysis for Multimedia Interactive Services (WIAMIS), 2010.

[3] Alireza Fathi, Maria Florina Balcan , Xiaofeng Ren,James M. Rehg,"Combining Self Training and Active Learning for Video Segmentation”, British Machine Vision Conference,2011.

[4] Çigdem Eroglu Erdem and Bülent Sankur, "Performance evaluation metrics for object-based video segmentation", Proceeding of IEEE International Conference on Image Processing, 2001.

[5] Sohaib Khan and Mubarak Shah,” Object Based Segmentation of Video Using Color, Motion and Spatial Information", Proceedings of the Conference on IEEE Computer Society, vol.2, no.1, 2003.

[6] Kashinath Murmu Vineet Kumar," Wavelet Based Video Segmentation and Indexing",EE678 Wavelets Application Assignment, April 2005.

[7] Michal Haindl, Pavel Zid and Radek Holub,"Range video segmentation", Proceedings of the IEEE 10th International Conference on Information Science, Signal Processing and their Applications, 2010.

[8] Ram Kumar Yadav, Sanjeev Sharma and Jitendra Singh Verma,"Deformation and Improvement of Video Segmentation Based on morphology Using SSD Technique" IJCTA, Vol. 2, no. 5, pp.13221327, 2011.

[9] Jose Lezama, Karteek Alahari, Josef Sivic and Ivan Laptev, "Track to the Future: Spatio-temporal Video Segmentation with Long-range Motion Cues", In Proceedings of the IEEE Conference on Computer Vision and Pattern Recognition (CVPR), 2011.

[10] "Exploiting Nonlocal Spatiotemporal Structure for Video Segmentation",https://wiki.engr.illinois.edu/.../report_video_segmentation.pdf?.

[11] Yining Deng and B. S. Manjunath, "Unsupervised Segmentation of Color-Texture Regions in Images and Video", IEEE Transactions on Pattern Analysis and Machine Intelligence, vol.23, no.8, 2002.

[12] Yasira Beevi C and S. Natarajan, "An efficient Video Segmentation Algorithm with Real time Adaptive Threshold Technique", International Journal of Signal Processing, Image Processing and Pattern Recognition Vol. 2, no.4, December 2009.

[13] Si Wu, Yu-Fei Ma, Hong-Jiang Zhang, "Video quality classification based home video segmentation" IEEE, 2005.

[14] N. Peyrard and P. Bouthemy, "Content-Based Video Segmentation using Statistical Motion Models", In Proceeding of British Machine Vision conference, 2002.

[15] Hongtu Jiang, Hakan Ardo and Viktor Owall, "Hardware Accelerator Design for Video Segmentation with Multi-modal Background Modeling", IEEE, 2005.

[16] Cheng Chen and Guoliang Fan, "Perception Principles Guided Video Segmentation".

[17] Yong Jae Lee,Jaechul Kim, and Kristen Grauman, "Key-SegmentsforVideoObjectSegmentation", Proceedings of the International Conference on Computer Vision (ICCV), 2011. 